\title{
Lack of Detection of Xenotropic Murine Leukemia Virus-Related Virus in HIV-1 Lymphoma Patients
}

\author{
Krista A. Delviks-Frankenberry, ${ }^{1}$ Chawaree Chaipan, ${ }^{1}$ Rachel Bagni, ${ }^{2}$ \\ Kathleen Wyvill, ${ }^{3}$ Robert Yarchoan, ${ }^{3}$ and Vinay K. Pathak ${ }^{1}$ \\ ${ }^{1}$ Viral Mutation Section, HIV Drug Resistance Program, National Cancer Institute at Frederick, \\ National Institutes of Health, Frederick, MD 21702, USA \\ ${ }^{2}$ Protein Expression Laboratory, SAIC-Frederick Inc., NCI-Frederick, Frederick, MD 21702, USA \\ ${ }^{3}$ HIV and AIDS Malignancy Branch, National Cancer Institute, National Institutes of Health, \\ Bethesda, MD 20892, USA
}

Correspondence should be addressed to Vinay K. Pathak, vinay.pathak@nih.gov

Received 17 June 2011; Accepted 11 July 2011

Academic Editor: Yoshinao Kubo

Copyright () 2011 Krista A. Delviks-Frankenberry et al. This is an open access article distributed under the Creative Commons Attribution License, which permits unrestricted use, distribution, and reproduction in any medium, provided the original work is properly cited.

\begin{abstract}
Xenotropic murine leukemia virus-related virus (XMRV) is a gammaretrovirus reported to be associated with human prostate cancer and chronic fatigue syndrome. Since retroviruses cause various cancers, and XMRV replication might be facilitated by HIV1 co-infection, we asked whether certain patients with HIV-associated lymphomas are infected with XMRV. Analysis of PMBCs and plasma from 26 patients failed to detect XMRV by PCR, ELISA, or Western blot, suggesting a lack of association between XMRV and AIDS-associated lymphomas.
\end{abstract}

\section{Introduction}

A gammaretrovirus, xenotropic murine leukemia virusrelated virus (XMRV), was recently discovered and reported to be associated with human prostate cancer (PC) [1]. In the initial report in PC patients, there was a strong correlation between detection of XMRV and a genetic defect in the innate immunity gene RNASEL [1]. However, subsequent studies in PC patients showed either no such association [2] or little or no evidence of XMRV infection (reviewed in [3]). Interest in XMRV was increased by the finding that a high percentage of patients with chronic fatigue syndrome (CFS) as well as some asymptomatic patients were infected with XMRV [4]. However, other studies have failed to find such an association (reviewed in [3]). XMRV has been shown to infect peripheral blood mononuclear cells (PBMCs) and CD4+ $\mathrm{T}$ cells in vitro, indicating that XMRV can infect the same target cells as HIV-1 ([4] and data not shown). However, our recent studies showed that productive replication of XMRV in PBMC and spread in culture are severely restricted by APOBEC 3 proteins and perhaps other host defense mechanisms [5]. It remained possible, however, that target cells infected with HIV1 might provide a favorable environment for XMRV to replicate by depleting cellular host restriction factors such as APOBEC3G, known to inhibit XMRV infection $[6,7]$. Several factors led us to explore the possibility that certain patients with HIV-associated lymphoma might be infected with XMRV. Gammaretroviruses can cause lymphomas in other species (reviewed in [8]), and it has been postulated that a number of cases of HIV-associated lymphomas might be caused by an as-yet-unidentified virus [9]. It is also important to note that patients with HIV infection have a higher prevalence of infection with other viruses, and their immunocompromised state might permit more efficient replication of other viruses, including XMRV [9]. Because of the high degree of concern regarding potential XMRV infection and spread in the human population, we sought to investigate whether XMRV might be present in a subset of patients with HIV-associated lymphomas. 
TABle 1: HIV-1 Lymphoma Patient Cohort Characteristics.

\begin{tabular}{|c|c|}
\hline No. of patients & 26 \\
\hline Sex, male/female & $24 / 2$ \\
\hline Age, median years (range) & $38(21-58)$ \\
\hline $\begin{array}{l}\text { CD } 4 \text { count, cells } / \mathrm{mm}^{3} \text {, } \\
\text { median (range) }\end{array}$ & $76(0-713)$ \\
\hline $\begin{array}{l}\text { HIV viremic }{ }^{\text {a }} \text { No. } \\
\text { postitive/No. tested }\end{array}$ & $12 / 21$ \\
\hline On anti-HIV drugs & $24 / 26$ \\
\hline On AZT & $14 / 26$ \\
\hline \multirow[t]{8}{*}{$\begin{array}{l}\text { Lymphomas studied (No. } \\
\text { of patients): }\end{array}$} & PCNSL $^{\mathrm{b}}(11)$ \\
\hline & $\begin{array}{l}\text { Diffuse large B cell } \\
\text { lymphoma }(8)\end{array}$ \\
\hline & Burkitts (1) \\
\hline & Plasmablastic (1) \\
\hline & $\begin{array}{l}\text { Primary effusion } \\
\text { lymphoma (1) }\end{array}$ \\
\hline & Primary intraocular (1) \\
\hline & Hodgkin lymphoma (2) \\
\hline & $\begin{array}{l}\text { Head mass- presumptive } \\
\text { PCNSL (1) }\end{array}$ \\
\hline
\end{tabular}

${ }^{a}$ Detectable p24 Ag or HIV RNA.

${ }^{b}$ Primary Central Nervous System Lymphoma.

\section{Analysis and Results}

Total nucleic acids from PBMCs were isolated (Qiagen DNA Blood Mini Kit) from 26 HIV-1 infected patients previously diagnosed with AIDS-associated lymphomas (Table 1). The study was approved by the National Cancer Institutional Review Board, and all patients and donors gave written informed consent. Using a real-time quantitative PCR (qPCR) assay that employed primers specific for a unique 24nucleotide gap in XMRV gag (primer and probe sequences as reported in, [10]), $500 \mathrm{ng}$ of patient DNA was tested in each reaction using the Roche LightCycler 480 Probes Master mix. Cycling conditions using the LightCycler 480 Roche instrument (Roche Diagnostics) were $95^{\circ} \mathrm{C}$ for $30 \mathrm{sec}$ followed by 50 cycles at $95^{\circ} \mathrm{C}$ for $15 \mathrm{sec}$ and $60^{\circ} \mathrm{C}$ for $60 \mathrm{sec}$. Genomic DNA from the XMRV-expressing cell line $22 \mathrm{R} v 1$ (ATCC) was used to generate a standard curve for XMRV ( 20 proviral copies/cell). The standard curve was spiked with $500 \mathrm{ng}$ 293T DNA to ensure similar amplification efficiencies as the patient samples. The qPCR had detection sensitivity of $<5$ XMRV copies/75,757 cells. The quality and quantity of input DNA were confirmed by detection of human GAPDH by qPCR. Using this assay, all 26 HIV1 lymphoma patients tested were negative for XMRV gag sequences (Figure 1).

Patient plasma was also screened by ELISA (Bagni, Protein Expression Laboratory, SAIC-Frederick Inc., NCIFrederick, unpublished results) for antibodies against XMRV capsid (CA) and envelope (transmembrane, TM) proteins (Figure 2). Due to the absence of definitive XMRV positive patient control samples, sera from macaques before and after

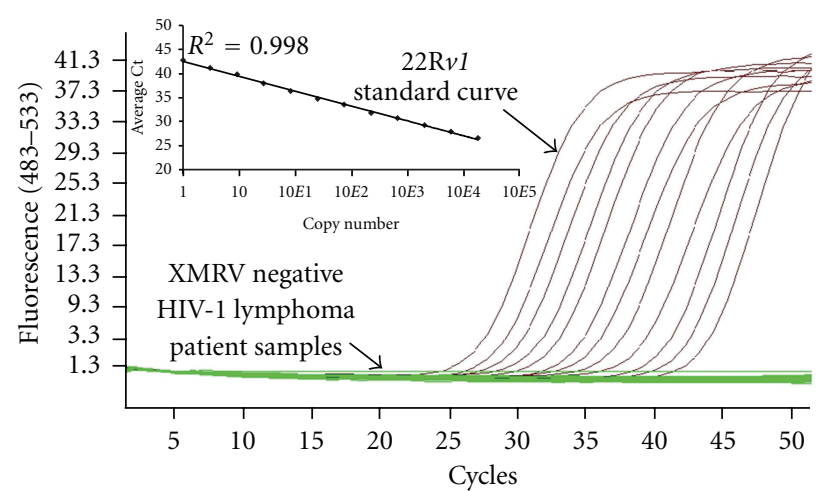

FIgure 1: Real-time PCR analysis of HIV-1 lymphoma positive patients for XMRV. XMRV gag qPCR failed to detect XMRV from DNA isolated from PBMCs for the 26 HIV-1 lymphoma patients (green lines). Inset shows single-copy sensitivity of the assay.

experimental XMRV infection served as controls for baseline and positive reactivity (Lifson and Del Prete, AIDS and Cancer Virus Program, SAIC-Frederick, Inc., unpublished results). Briefly, plasma samples were collected before and after-inoculation (119 days) from two pigtail macaques inoculated with $4.8 \times 10^{9}$ XMRV RNA equivalents derived from 22R $v 1$ cell supernatants (Lot SP1592, Biological Products Core, AIDS and Cancer Virus Program, SAIC Frederick, Inc., NCI-Frederick). Samples were considered reactive if they were at least $50 \%$ reactive relative to the macaque samples following XMRV infection (positive control sera). None of the 26 HIV-1 lymphoma patient, nor the 10 healthy donors, tested positive for the CA (Figure 2(a)) or TM protein (Figure 2(b)), although 2/26 patients had slight reactivity to TM (asterisk, Figure 2(b)). To evaluate these 2 patients, Western blot analysis was performed to assess whether the TM-reactive ELISA test reflected an immune response to XMRV. Endpoint dilution analysis indicated that a $1: 2000$ dilution of the positive macaque sera produced reproducible positive bands on the western blot with a high signal to noise ratio (Lifson and Del Prete, AIDS and Cancer Virus Program, SAIC-Frederick, Inc., unpublished results). Sera from patients (diluted $1: 50$ or $1: 200$ ) and positive sera from macaques (diluted $1: 2000$ ) were tested against XMRV viral lysates obtained from the $22 \mathrm{R} v 1$ cell line. The sera from both TM-reactive patients (10- to 40-fold more concentrated than the macaque) failed to detect capsid, TM, or other XMRV proteins (data not shown), indicating that the TMpositive ELISA test was most likely due to the presence of crossreactive nonspecific antibodies.

\section{Discussion}

Our studies show a lack of association between XMRV and AIDS-associated lymphomas and complement other studies that have failed to detect XMRV in the PBMCs, plasma, or seminal plasma from HIV-1 infected patients [11-16]. A potential link between XMRV and cancer is hypothesized in that gammaretroviruses can cause sarcomas and leukemias in rodents, felines, and primates (reviewed 


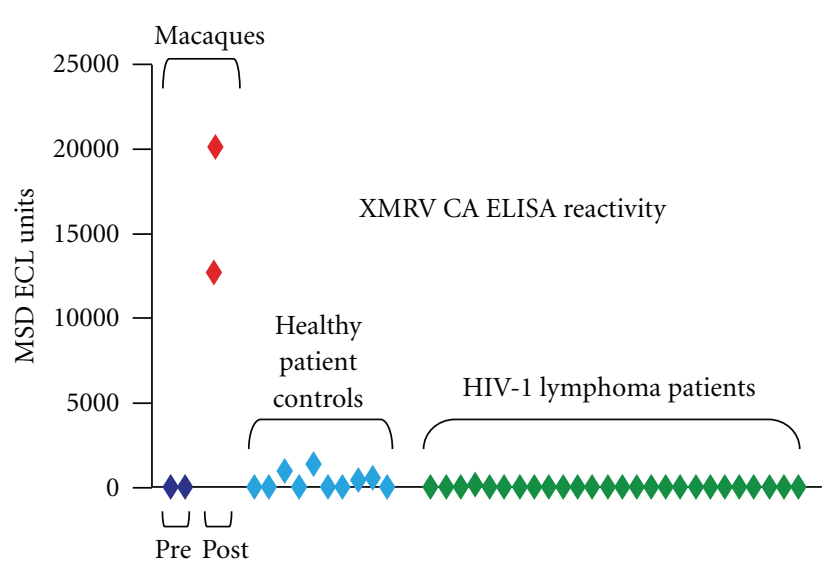

(a)

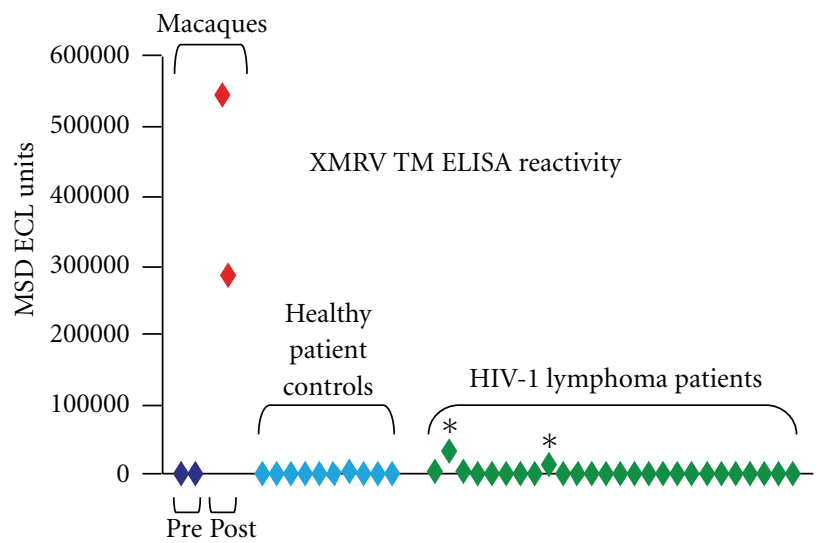

(b)

FIGURe 2: Patient reactivity to XMRV proteins CA and TM. Plasma from the $26 \mathrm{HIV}-1$ lymphoma positive patients (green diamonds) were assayed by ELISA versus plasma from ten healthy donor controls (light blue diamonds) and two Macaques infected with XMRV pre- (dark blue diamonds) and post- (red diamonds) infection. ${ }^{*}$ indicates two patients with minimal TM reactivity.

in [8]). However, it is unclear whether XMRV infection is associated with prostate or other human cancers. A survey of 800 and 539 PC samples from the United States and Germany, respectively, showed no association with XMRV $[17,18]$. A survey of 134 prostate cancer patient plasma samples by us and our collaborators also failed to detect any evidence of XMRV (Kearney et al., under review for this issue of Advances in Virology). Several recent studies have reported that contamination of human samples with mouse DNA may have contributed to XMRV's reported association with human disease [19-22]. Garson et al. [23] also reported that two integration site sequences, previously shown to demonstrate XMRV integration into patient DNA, likely were the result of contamination of identical integration sites from infected DU145 cells reported by the same lab. Furthermore, our recent studies provide compelling evidence that XMRV was generated through recombination of two endogenous murine leukemia viruses during the passage of a PC xenograft in nude mice, and therefore all detection of XMRV from human samples are likely to be due to contamination that originated from this recombination event [24]. It has been hypothesized that this lab-derived virus may have escaped from the lab and is now circulating in the human population. However, our failure to detect XMRV in HIV-1-associated lymphoma patients, along with $>10$ other studies of different patient cohorts performed by independent investigators, argues against this possibility.

In Summary, we were unable to detect XMRV DNA or XMRV-specific antibodies in the PBMCs or plasma from HIV-1-associated lymphoma patients, further supporting the absence of a link between XMRV and human cancer.

\section{Acknowledgments}

The authors thank Wei-Shau Hu for valuable input throughout the project and critical reading of the manuscript and Katie Beam and Allison Meade for technical assistance.
The content of this publication does not necessarily reflect the views or policies of the Department of Health and Human Services nor does mention of trade names, commercial products, or organizations imply endorsement by the U.S. Government. The funders had no role in study design, data collection and analysis, decision to publish, or preparation of the manuscript. This work was supported in part by the Intramural Research Program of the National Institutes of Health, National Cancer Institute, Center for Cancer Research, and a Bench-to-Bedside Award to Vinay K. Pathak.

\section{References}

[1] A. Urisman, R. J. Molinaro, N. Fischer et al., "Identification of a novel gammaretrovirus in prostate tumors of patients homozygous for R462Q RNASEL variant," PLoS Pathogens, vol. 2, no. 3 article e25, pp. 211-225, 2006.

[2] R. Schlaberg, D. J. Choe, K. R. Brown, H. M. Thaker, and I. R. Singh, "XMRV is present in malignant prostatic epithelium and is associated with prostate cancer, especially high-grade tumors," Proceedings of the National Academy of Sciences of the United States of America, vol. 106, no. 38, pp. 16351-16356, 2009.

[3] G. Simmons, S. A. Glynn, J. A. Holmberg et al., "The Blood Xenotropic Murine Leukemia Virus-Related Virus Scientific Research Working Group: mission, progress, and plans," Transfusion, vol. 51, no. 3, pp. 643-653, 2011.

[4] V. C. Lombardi, F. W. Ruscetti, J. D. Gupta et al., "Detection of an infectious retrovirus, XMRV, in blood cells of patients with chronic fatigue syndrome," Science, vol. 326, no. 5952, pp. 585-589, 2009.

[5] C. Chaipan, K. A. Dilley, T. Paprotka et al., "Severe restriction of xenotropic murine leukemia virus-relate virus replication and spread in cultured human peripheral blood mononuclear cells," Journal of Virology, vol. 85, no. 10, pp. 4888-4897, 2011.

[6] T. Paprotka, N. J. Venkatachari, C. Chaipan et al., "Inhibition of xenotropic murine leukemia virus-related virus by APOBEC3 proteins and antiviral drugs," Journal of Virology, vol. 84, no. 11, pp. 5719-5729, 2010. 
[7] H. C. T. Groom, M. W. Yap, R. P. Galão, S. J. D. Neil, and K. N. Bishop, "Susceptibility of xenotropic murine leukemia virus-related virus (XMRV) to retroviral restriction factors," Proceedings of the National Academy of Sciences of the United States of America, vol. 107, no. 11, pp. 5166-5171, 2010.

[8] N. Maeda, H. Fan, and Y. Yoshikai, "Oncogenesis by retroviruses: old and new paradigms," Reviews in Medical Virology, vol. 18, no. 6, pp. 387-405, 2008.

[9] R. Yarchoan, G. Tosato, and R. F. Little, "Therapy insight: AIDS-related malignancies-the influence of antiviral therapy on pathogenesis and management," Nature Clinical Practice Oncology, vol. 2, no. 8, pp. 406-415, 2005.

[10] S. Hong, E. A. Klein, J. Das Gupta et al., "Fibrils of prostatic acid phosphatase fragments boost infections with XMRV (Xenotropic Murine leukemia virus-Related Virus), a human retrovirus associated with prostate cancer," Journal of Virology, vol. 83, no. 14, pp. 6995-7003, 2009.

[11] K. J. Kunstman, T. Bhattacharya, J. Flaherty, J. P. Phair, and S. M. Wolinsky, "Absence of xenotropic murine leukemia virusrelated virus in blood cells of men at risk for and infected with HIV," AIDS, vol. 24, no. 11, pp. 1784-1785, 2010.

[12] S. Tang, J. Zhao, R. Viswanath et al., "Absence of detectable xenotropic murine leukemia virus-related virus in plasma or peripheral blood mononuclear cells of human immunodeficiency virus Type 1-infected blood donors or individuals in Africa," Transfusion, vol. 51, no. 3, pp. 463-468, 2011.

[13] M. Cornelissen, F. Zorgdrager, P. Blom et al., "Lack of detection of XMRV in seminal plasma from HIV-1 infected men in The Netherlands," PLoS ONE, vol. 5, no. 8, Article ID e12040, 2010.

[14] E. Barnes, P. Flanagan, A. Brown et al., "Failure to detect xenotropic murine leukemia virus-related virus in blood of individuals at high risk of blood-borne viral infections," Journal of Infectious Diseases, vol. 202, no. 10, pp. 1482-1485, 2010.

[15] T. J. Henrich, J. Z. Li, D. Felsenstein et al., "Xenotropic murine leukemia virus-related virus prevalence in patients with chronic fatigue syndrome or chronic immunomodulatory conditions," Journal of Infectious Diseases, vol. 202, no. 10, pp. 1478-1481, 2010.

[16] E. R. Gray, J. A. Garson, J. Breuer et al., "No evidence of XMRV or related retroviruses in a london HIV-1-positive patient cohort," PLoS ONE, vol. 6, no. 3, 2011.

[17] A. L. Aloia, K. S. Sfanos, W. B. Isaacs et al., "XMRV: a new virus in prostate cancer?” Cancer Research, vol. 70, no. 24, pp. 10028-10033, 2010.

[18] O. Hohn, H. Krause, P. Barbarotto et al., "Lack of evidence for xenotropic murine leukemia virus-related virus(XMRV) in German prostate cancer patients," Retrovirology, vol. 6, article no. 1742, p. 92, 2009.

[19] S. Hue, E. R. Gray, A. Gall et al., "Disease-associated XMRV sequences are consistent with laboratory contamination," Retrovirology, vol. 7, no. 1 article 111, 2010.

[20] B. Oakes, A. K. Tai, O. Cingoz et al., "Contamination of human DNA samples with mouse DNA can lead to false detection of XMRV-like sequences," Retrovirology, vol. 7, article 109, 2010.

[21] M. J. Robinson, O. W. Erlwein, S. Kaye et al., "Mouse DNA contamination in human tissue tested for XMRV," Retrovirology, vol. 7, article 108, 2010.

[22] E. Sato, R. A. Furuta, and T. Miyazawa, "An endogenous murine leukemia viral genome contaminant in a commercial RT-PCR Kit is amplified using standard primers for XMRV," Retrovirology, vol. 7, article 110, 2010.
[23] J. A. Garson, P. Kellam, and G. J. Towers, "Analysis of XMRV integration sites from human prostate cancer tissues suggests PCR contamination rather than genuine human infection," Retrovirology, vol. 8, article 13, 2011.

[24] T. Paprotka, K. A. Delviks-Frankenberry, O. Cingöz et al., "Recombinant Origin of the Retrovirus XMRV," Science, vol. 333, no. 6038, pp. 97-101, 2011. 

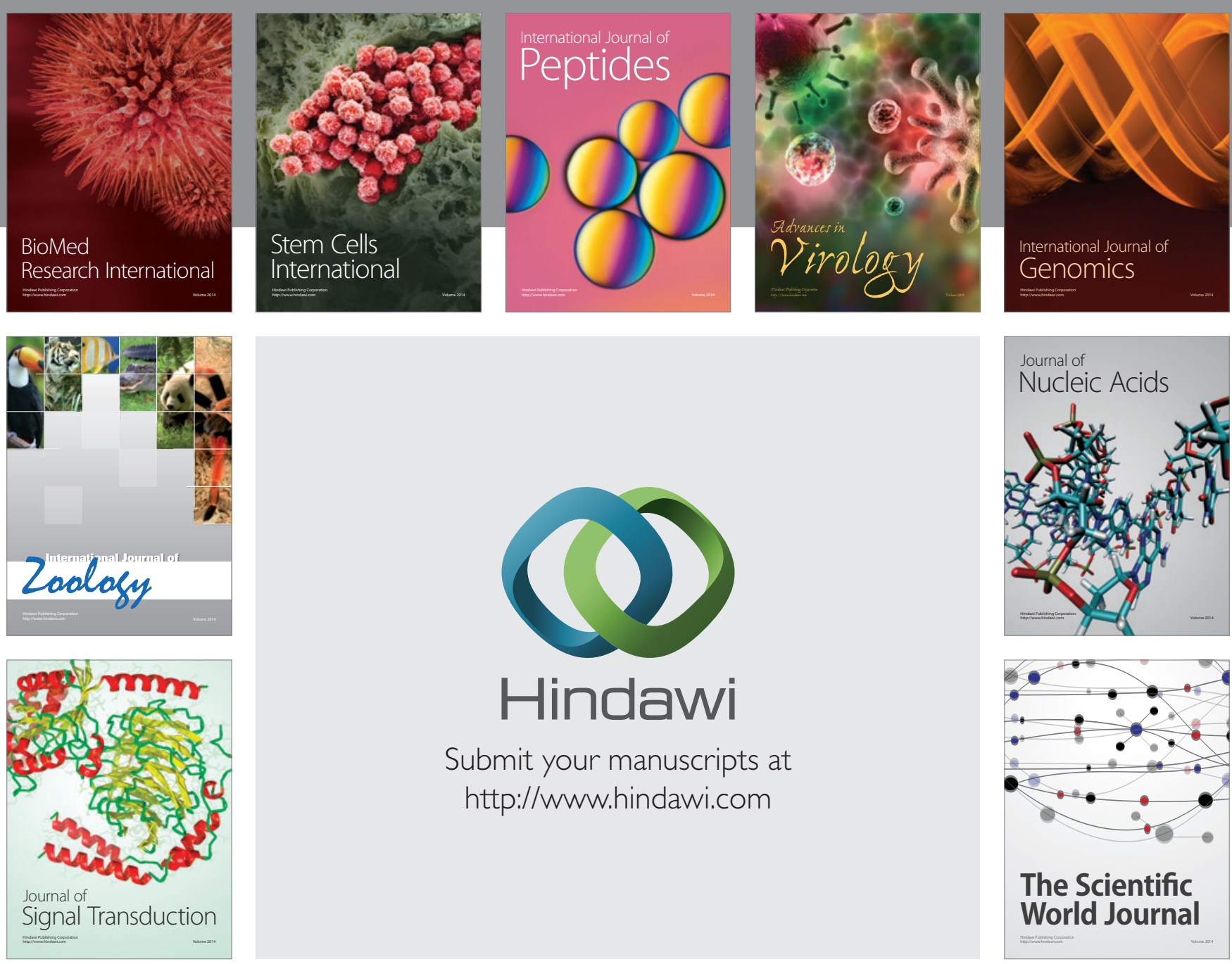

Submit your manuscripts at

http://www.hindawi.com
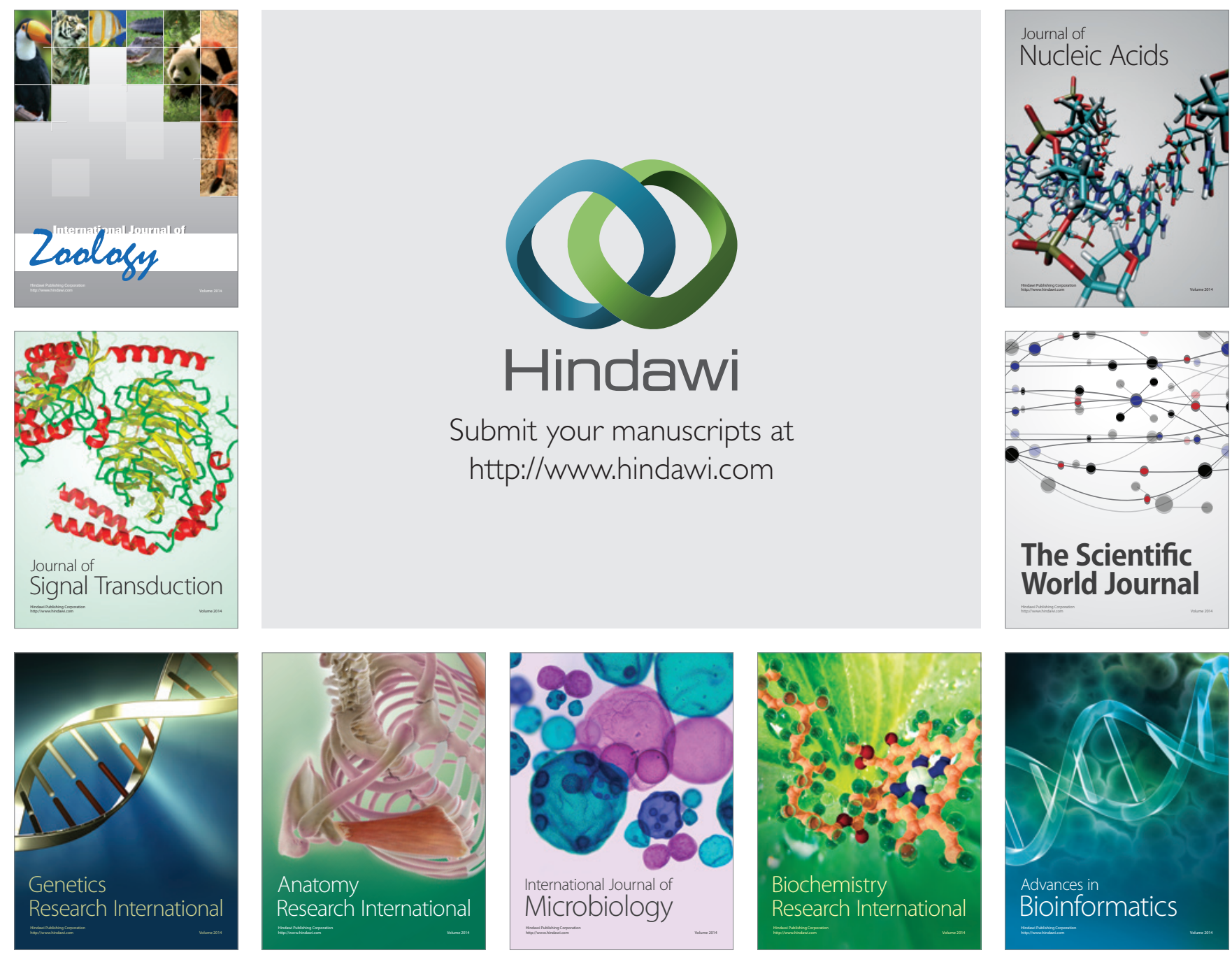

The Scientific World Journal
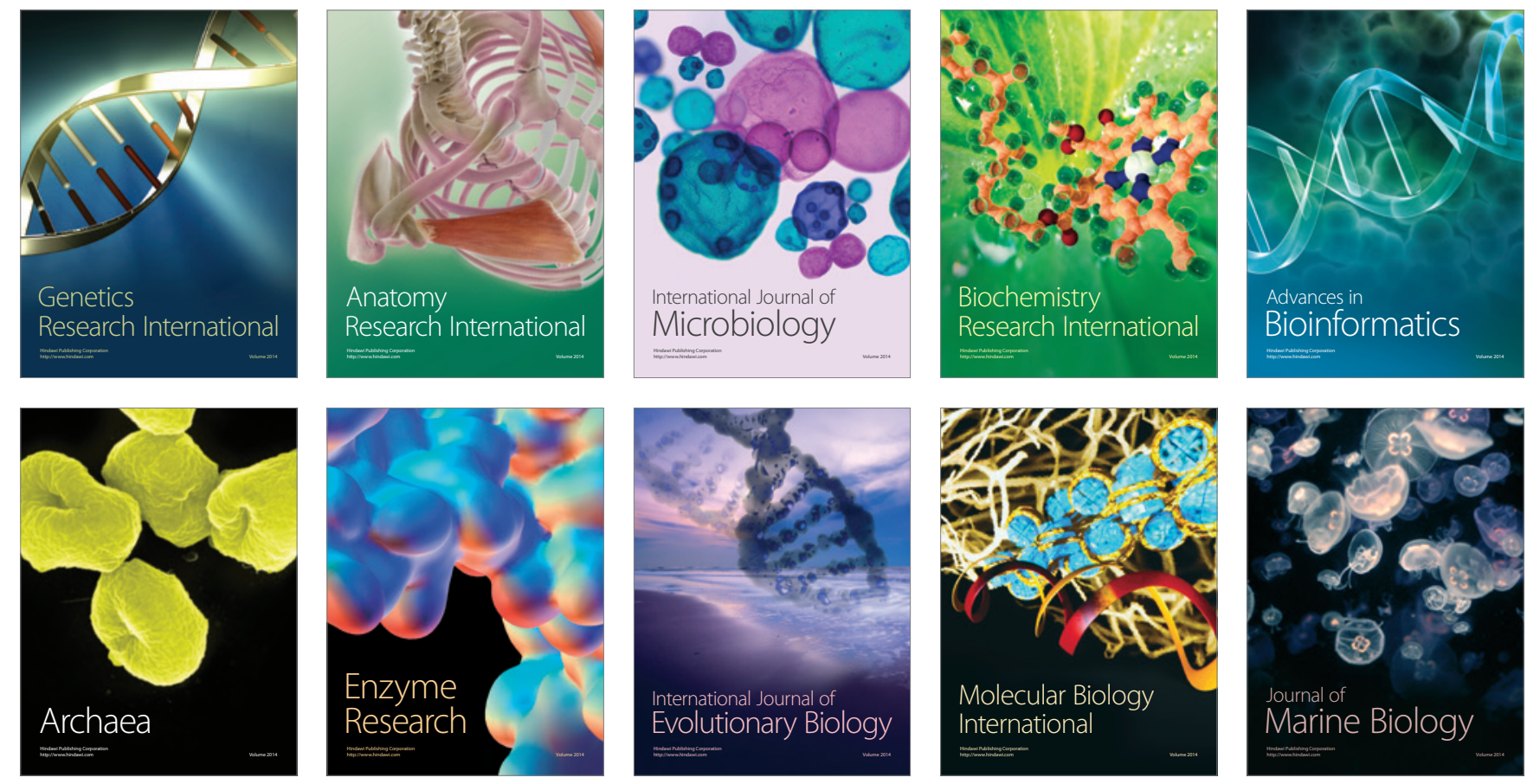